\title{
Suzaku observations of the low surface brightness cluster A76
}

\author{
N. Ota ${ }^{1}$, Y. Fujino ${ }^{1}$, Y. Ibaraki ${ }^{1}$, H. Böhringer ${ }^{2}$, and G. Chon ${ }^{2}$ \\ 1 Department of Physics, Nara Women's University, Kitauoyanishimachi, Nara, 630-8501 Nara, Japan \\ e-mail: naomi@cc.nara-wu.ac.jp \\ 2 Max-Planck-Institut für extraterrestrische Physik, Giessenbachstraße, 85748 Garching, Germany
}

Received 10 April 2013 / Accepted 19 May 2013

\begin{abstract}
Aims. We present the results of Suzaku observations of a nearby galaxy cluster, A76 at $z=0.0395$. This cluster is characterized by extremely low X-ray surface brightness and is hereafter referred to as the LSB cluster. We investigate the nature and thermodynamic evolution of the LSB cluster by studying the physical properties of the hot intracluster medium in A76.

Methods. We conducted two-pointed Suzaku observations of A76 and examined the global gas properties of the cluster by XIS spectral analysis. We also performed deprojection analysis of annular spectra and derived radial profiles of gas temperature, density, and entropy out to approximately $850 \mathrm{kpc}\left(\sim 0.6 r_{200}\right)$ in A76 East and $560 \mathrm{kpc}\left(\sim 0.4 r_{200}\right)$ in A76 West.

Results. The measured global temperature and metal abundance are approximately $3.3 \mathrm{keV}$ and 0.24 solar, respectively. From the deprojection analysis, the entropy profile is found to be flat with respect to radius. The entropy within the central region $\left(r<0.2 r_{200}\right)$ is exceptionally high $\left.(\sim 400 \mathrm{keV} \mathrm{cm})^{2}\right)$, a phenomenon that is not readily explained by either gravitational heating or preheating. The X-ray morphology is clumped and irregular, and the electron density is extremely low $\left(10^{-4}-10^{-3} \mathrm{~cm}^{-3}\right)$ for the observed high temperature, suggesting that A76 is in the early phase of cluster formation and that the gas compression due to gravitational potential confinement is lagging behind the gas heating.
\end{abstract}

Key words. galaxies: clusters: individual: A76 - galaxies: clusters: intracluster medium - X-rays: galaxies: clusters cosmology: observations

\section{Introduction}

With the exception of rare dramatic cluster mergers, galaxy clusters feature closely self-similar X-ray surface brightness profiles, with a steep decrease with radius (e.g., Ota \& Mitsuda 2004; Croston et al. 2008). A small fraction of clusters (5-10\%) exhibit very low surface brightness distribution and highly diffuse X-ray emission, as found, e.g., in cluster samples detected in the ROSAT All-Sky Survey (RASS; Böhringer et al. 2001). Among representative subsamples of galaxy clusters from the RASS-based REFLEX survey of 33 objects (the REXCESS project; Böhringer et al. 2007), three such LSB clusters have been identified.

A possible interpretation of this characteristic is that the objects are dynamically very young. In most clusters, the cluster core region presumably forms first, and subsequently, a large cluster mass accretes. In contrast, these objects may develop from rarer overdensities, which more closely resemble overdense homogeneous spheres. Such objects would show a nearly homogeneous collapse with a high degree of local clumping.

This scenario can be validated from the entropy distribution of the intracluster medium (ICM) in these cluster systems (e.g., Voit 2005). The three LSB clusters in the REXCESS sample are distinguished by high $\left(\gtrsim 200 \mathrm{keV} \mathrm{cm}^{2}\right)$ central entropy. This is puzzling, since the above scenario requires an intermediate stage with very high central entropy that must be lowered again for normal cluster formation. Alternatively, we note that the temperature of the LSB clusters is higher than for normal clusters at any given gas density. When a cluster collapses, the deepening of its potential well is manifested by an increase in temperature (which approximately reflects the potential depth). The gas should be simultaneously compressed by potential confinement.
The very high entropy suggests that, by some unknown mechanism, the gas compression is lagging behind the heating of the gas. Alternatively, unexpected heating processes may have boosted the entropy and inflated the ICM in these systems.

To probe the nature of the LSB clusters and test the above scenarios further, we here focus on the extremely LSB galaxy cluster A76, which exhibits irregular morphology. Indeed, the surface brightness of A76 is the lowest among the ROSAT clusters studied in Neumann \& Arnaud (1999). The main immediate objective of these studies is to assess the temperature and entropy structure of the clusters using Suzaku satellite data (Mitsuda et al. 2007). The aim is to better understand the formation process and history of these objects.

In this study, the cosmological model is adopted with the matter density $\Omega_{\mathrm{M}}=0.27$, the cosmological constant $\Omega_{\Lambda}=0.73$, and the Hubble constant $H_{0}=70 \mathrm{~km} \mathrm{~s}^{-1} \mathrm{Mpc}^{-1}$. At the cluster redshift $(z=0.0395), 1^{\prime}$ corresponds to $47 \mathrm{kpc}$. Unless otherwise noted, specified errors indicate the $90 \%$ confidence intervals.

\section{Observation and data reduction}

Two-pointed observations of A76 (in the western and eastern directions) were conducted between 2009 Dec. 18 and 2009 Dec. 19 in the Suzaku AO-4 period. The details of the observations are summarized in Table 1. The XIS instruments consist of four X-ray sensitive CCD cameras: three frontilluminated (XIS-0, -2, -3) and one back-illuminated (XIS-1; Koyama et al. 2007). The XIS-0, -1 , and -3 CCD cameras were operated in normal mode with the space charge injection enabled (Uchiyama et al. 2009).

As shown in Fig. 1a, diffuse X-ray emission from the cluster is clearly detected by Suzaku/XIS. The X-ray peak position 

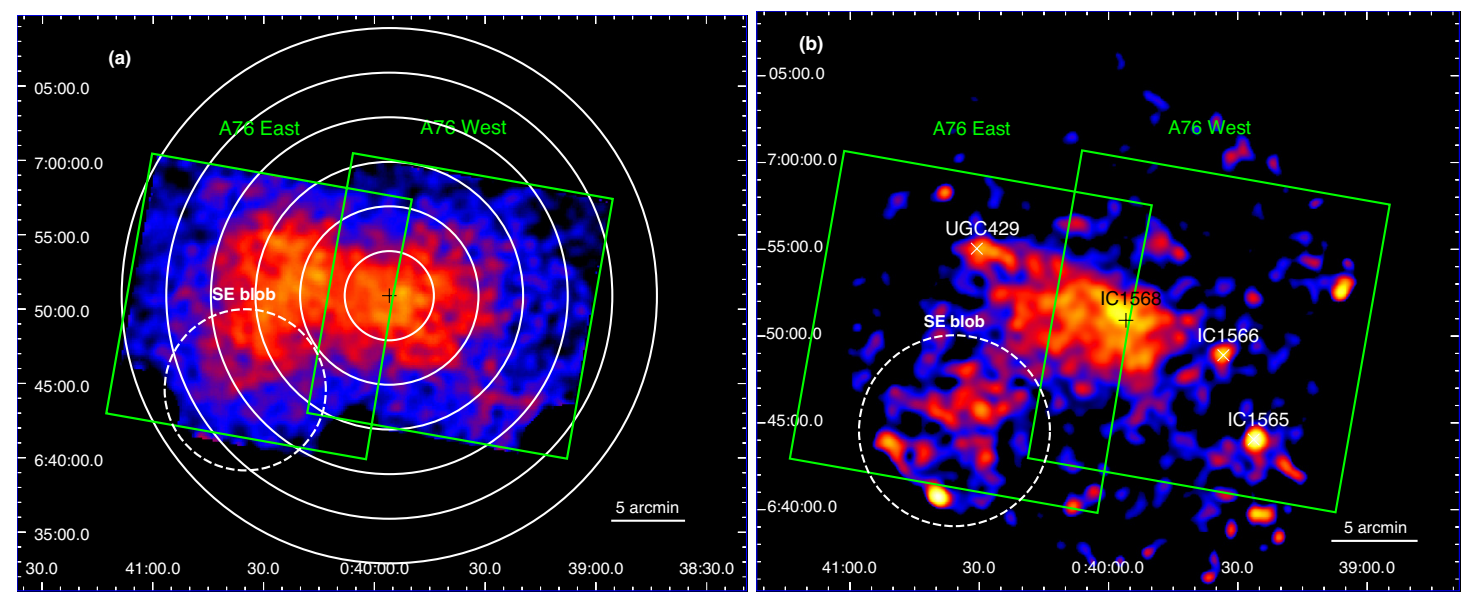

Fig. 1. a) Suzaku XIS-1 mosaic image of A76 in the $0.5-5 \mathrm{keV}$ band. The image is corrected for exposure map and vignetting effect and is smoothed by a Gaussian function with $\sigma=40^{\prime \prime}$, without background subtraction. The location of the X-ray peak corresponding to member galaxy IC 1568 is marked with the plus sign. The spectral integration regions used in Sects. 3.1 and 3.2 lie within the green boxes and white circles, respectively. The two corners of the CCD chip illuminated by ${ }^{55} \mathrm{Fe}$ calibration sources are excluded from the image. b) XMM-Newton PN image of A76 in the $0.5-2 \mathrm{keV}$ band with subtracted background and $\sigma=20^{\prime \prime}$ Gaussian smoothing. The net exposure time is approximately $600 \mathrm{~s}$. The positions of three detected sources (Sect. 2) and the SE blob (Sect. 4) are indicated by crosses and a dashed circle, respectively.

Table 1. Log of Suzaku observations of A76.

\begin{tabular}{lccccc}
\hline \hline Target & Obs ID & Date & \multicolumn{2}{c}{ Coordinates $^{a}$} & \multicolumn{2}{c}{ Exposure $^{b}$} \\
& & & RA & Dec & {$[\mathrm{s}]$} \\
\hline A76 East & 804087010 & 2009 Dec. 18 & $00: 40: 32.0$ & $06: 50: 14.6$ & 23640 \\
A76 West & 804088010 & 2009 Dec. 18, 19 & $00: 39: 36.7$ & $06: 50: 16.8$ & 15130 \\
\hline
\end{tabular}

Notes. ${ }^{(a)}$ Pointing coordinates in J2000. ${ }^{(b)}$ Net exposure time after data filtering.

coincides (within the attitude determination limits of Suzaku) with the optical coordinates of one of the member galaxies, IC 1568, (00:39:56.0, +06:50:54.9) in the J2000 coordinates (Uchiyama et al. 2008). Figure 1b shows the XMM-Newton/PN image of the cluster. The net exposure time after removing the periods of high background rates is about $600 \mathrm{~s}$. As is also evident from the image, the X-ray morphology is irregular and elongated along the western and southeastern directions.

Event files were created by pipeline processing version 2.4. Data were analyzed using HEAsoft version 6.12 and CALDB version 2012-09-02 for XIS and version 2011-06-30 for the X-ray telescopes (XRT; Serlemitsos et al. 2007). The XIS data were filtered according to the following criteria: the Earth elevation angle $>10^{\circ}$, day-Earth elevation angle $>20^{\circ}$, and satellite outside the South Atlantic Anomaly.

The cluster spectra were extracted from 1) the XIS full field of views and 2) annular regions centered on IC 1568. The global gas properties in both pointing regions were measured from the former spectrum, while the latter was used to investigate the radial distributions. In both cases, the two corners of the CCD chip covered by ${ }^{55} \mathrm{Fe}$ calibration sources, as well as circular regions with radius $1^{\prime}$ centered on three point sources detected in the XMM-Newton image (IC 1565, IC 1566, and UGC 429), were excluded from the integration regions. The non-X-ray background was subtracted using xisnxbgen (Tawa et al. 2008), while other background components, i.e., the cosmic X-ray background (CXB) and the Galactic emissions arising from the local hot bubble (LHB) and the Milky Way halo (MWH) were determined by using the same method described in Sakuma et al. (2011). In brief, the blank-sky data obtained from $93 \mathrm{ks}$ Lockman Hole observations (Obs ID 104002010) were modeled by the formula "apec ${ }_{\mathrm{LHB}}+\mathrm{wabs}^{*}\left(\operatorname{apec}_{\mathrm{MWH}}+\right.$ power-law $\left._{\mathrm{CXB}}\right)$ ". The derived parameters are listed in Table 2. The systematic error due to the positional dependence of the background is estimated to be $10 \%$ by comparing the Lockman hole spectra with the blank-sky spectra around ASAS J002511+1217.2 (OBSID 403039010), which is $\sim 6$ :6 offset from A76.

The energy response files were generated by using xisrmfgen. To account for the vignetting effect in the XRTs, and a decrease in the low-energy efficiency due to contaminants on the optical blocking filter of the XIS, the auxiliary response files were calculated using xissimar fgen (Ishisaki et al. 2007). Here the XMM-Newton image of A76 was used as the input surface brightness.

\section{Analysis and results}

\subsection{Global spectra}

To measure the average temperature of A76 East and West, we first analyzed the XIS spectra extracted from two $18^{\prime} \times$ $18^{\prime}$ square regions (solid boxes in Fig. 1). The observed 0.5-8 keV spectra of three sensors (XIS-0, XIS-1, and XIS-3) were simultaneously fitted to the APEC thermal plasma model (Smith et al. 2001). The redshift and Galactic hydrogen column density were fixed at $z=0.0395$ and $N_{\mathrm{H}}=3.4 \times 10^{20} \mathrm{~cm}^{-2}$ (LAB survey; Kalberla et al. 2005). For metal abundance, the tables in Anders \& Grevesse (1989) were used. The $\chi^{2}$ fitting was performed using XSPEC version 12.7. The XIS spectra are given in Fig. 2 and model parameters in Table 3. In both regions, the temperature and metal abundance are $k T \sim 3 \mathrm{keV}$ and $Z \sim 0.24$ solar with fractional errors of $4-5 \%$ and $17-35 \%$, respectively.

A simultaneous APEC-model fit to the A76 East and West spectra yields a global temperature $k T$ of $3.3 \pm 0.1 \mathrm{keV}$ $\left(\chi^{2} /\right.$ d.o.f. $\left.=662 / 569\right)$. From the relationship given in Table 2 

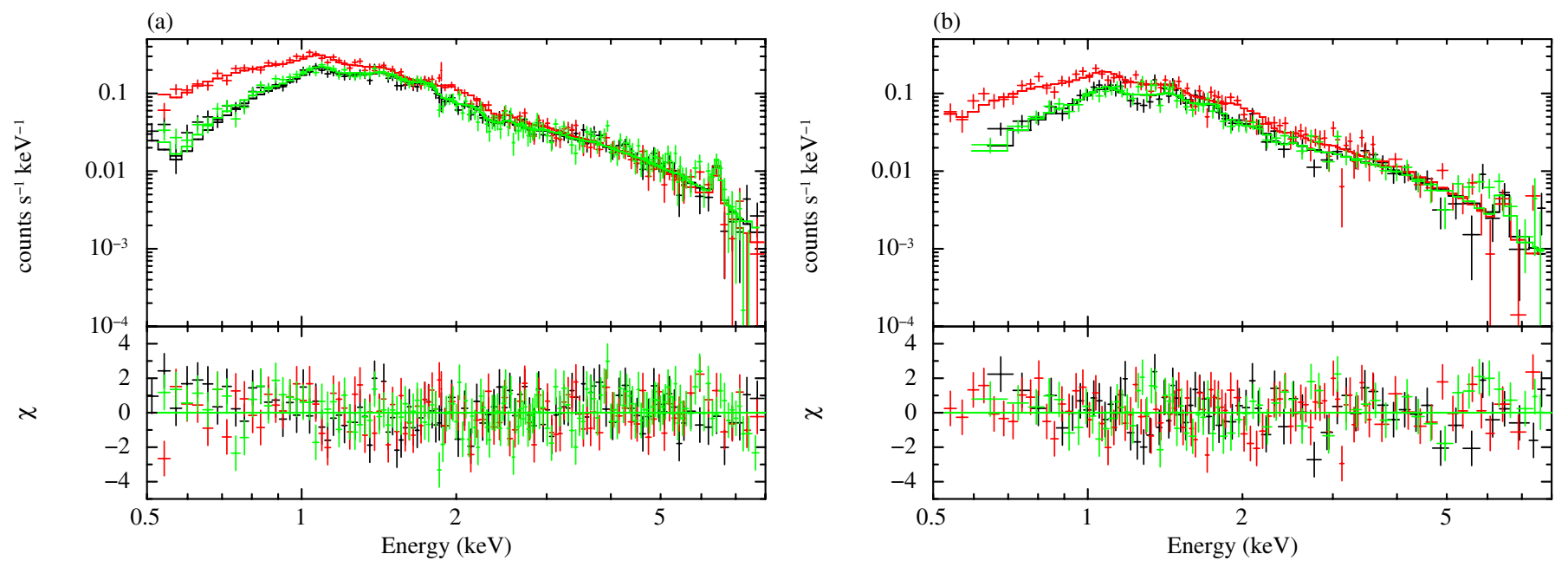

Fig. 2. Observed XIS spectra of a) A76 East and b) West. The spectra collected by XIS-0 (black), 1 (red), and 3 (green) are shown separately. The solid lines in the upper panel show the best-fitting APEC model simultaneously fitted to the three sensors convolved with the telescope and detector response functions. Bottom panel shows the residuals of the fit (in number of standard deviations).

Table 2. Spectral fits of the Lockman hole data for the CXB and Galactic components.

\begin{tabular}{lcccccc}
\hline \hline$\Gamma$ & Norm $^{a}$ & $\begin{array}{c}k T_{\mathrm{MWH}} \\
{[\mathrm{keV}]}\end{array}$ & Norm $_{\mathrm{MWH}}{ }^{b}$ & $\begin{array}{c}k T_{\mathrm{LHB}} \\
{[\mathrm{keV}]}\end{array}$ & Norm $_{\text {LHB }}$ & $\chi^{2} /$ d.o.f. \\
\hline $1.42_{-0.04}^{+0.04}$ & $1.05_{-0.04}^{+0.04} \times 10^{-3}$ & $0.27_{-0.05}^{+0.05}$ & $2.0_{-0.06}^{+0.11} \times 10^{-4}$ & $0.1(\mathrm{fix})$ & $2.63_{-0.07}^{+0.06} \times 10^{-3}$ & $820 / 704$ \\
\hline
\end{tabular}

Notes. ${ }^{(a)}$ The units are photons $\mathrm{keV}^{-1} \mathrm{~cm}^{-2} \mathrm{~s}^{-1}$ at $1 \mathrm{keV} .{ }^{(b)}$ Normalization of the APEC model, Norm $=\int n_{\mathrm{e}} n_{\mathrm{H}} \mathrm{d} V /\left(4 \pi(1+z)^{2} D_{\mathrm{A}}^{2}\right)\left[10^{-14} \mathrm{~cm}^{-5}\right]$. $D_{\mathrm{A}}$ is the angular diameter distance to the source. An $r=20^{\prime}$ uniform sky is assumed.

Table 3. APEC model parameters for spectra in A76 East and West.

\begin{tabular}{lcccc}
\hline \hline Region & $k T[\mathrm{keV}]$ & $Z$ [solar] & Norm $^{a}$ & $\chi^{2} /$ d.o.f. \\
\hline A76 East & $3.39_{-0.10}^{+0.15}$ & $0.24_{-0.04}^{+0.04}$ & $1.08_{-0.03}^{+0.03} \times 10^{-2}$ & $384 / 353$ \\
A76 West & $3.10_{-0.17}^{+0.17}$ & $0.23_{-0.07}^{+0.08}$ & $1.29_{-0.07}^{+0.08} \times 10^{-2}$ & $259 / 219$ \\
\hline
\end{tabular}

Notes. ${ }^{(a)}$ Normalization of the APEC model for the cluster emission.

of Arnaud et al. (2005) and the mean cluster temperature of $k T=3.3 \mathrm{keV}$, the viral radius is derived as $r_{200}=1.32 \mathrm{Mpc}$.

\subsection{Annular spectra}

To derive the radial temperature profile, we extracted spectra from $3^{\prime}$-wide concentric rings; $0^{\prime}-3^{\prime}, 3^{\prime}-6^{\prime}, 6^{\prime}-9^{\prime}, 9^{\prime}-12^{\prime}$, $12^{\prime}-15^{\prime}$, and $15^{\prime}-18^{\prime}$, as shown in Fig. 1. Because the X-ray image is asymmetric, the data from A76 East and West were separately analyzed. Note that the 6th ring $\left(15^{\prime}-18^{\prime}\right)$ is beyond the range of A76 West and is used only in A76 East. The XIS-0, 1,3 spectra of each ring were then simultaneously fitted to the APEC model in the same manner as that mentioned in Sect. 3.1. The best-fit parameters are listed in Table 4 .

The spectra of the innermost ring $\left(0^{\prime}<r<3^{\prime}\right)$ were derived from the A76 West data alone, since the A76 East data cover only half of the area. In the spectra of the outer rings $\left(15^{\prime}<\right.$ $r<18^{\prime}$ in A76 East and $12^{\prime}<r<15^{\prime}$ in A76 West), the metal abundance was fixed at its mean value ( 0.24 solar) because of large statistical uncertainty.

By summing the flux from all rings within $r<18^{\prime}$, the absorption-corrected flux and luminosity within $r<0.6 r_{200}$ in the $0.5-8 \mathrm{keV}$ are estimated to be $1.8 \times 10^{-11} \mathrm{erg} \mathrm{s}^{-1} \mathrm{~cm}^{-2}$ and $6.7 \times 10^{43} \mathrm{erg} \mathrm{s}^{-1}$, respectively. The bolometric luminosity of the cluster is $8.2 \times 10^{43} \mathrm{erg} \mathrm{s}^{-1}\left(r<0.6 r_{200}\right)$, approximately $1 / 3$ of the luminosity expected from the observed temperature and luminosity-temperature relationships of nearby clusters (e.g., Pratt et al. 2009).

\subsection{Deprojection analysis}

To further derive the three-dimensional structure of the gas density and entropy in the cluster, we performed a deprojection analysis by assuming spherical symmetry of the cluster gas distribution. Since the X-ray morphology of A76 is clearly irregular, this assumption introduces a systematic uncertainty into the analysis, which is examined below.

The APEC model corrected for the Galactic absorption was fitted to each radial bin, assuming that a metal abundance is radially constant. The arithmetic deprojection operation was performed by the "projet" model in XSPEC. The results of spectral fitting for A76 East and West are shown in Fig. 3 and Table 5. The spectra of $12^{\prime}<r<15^{\prime}$ were excluded from the analysis of A76 West because of poor statistics, which prevented convergence of model fit to the data. We confirmed that inclusion or exclusion of these outer spectra does not significantly affect the spectral parameters obtained for the remaining regions. In addition, since the uncertainty in the fitted temperature is large in the region $6^{\prime}<r<9^{\prime}$, the temperature of that region is linked to that of the inner region $\left(3^{\prime}<r<6^{\prime}\right)$.

The derived temperature profile is shown in Fig. 4a. The radius is normalized by $r_{200}$, and the electron density is calculated from the APEC normalization factor $\int n_{\mathrm{e}} n_{\mathrm{H}} \mathrm{d} V /(4 \pi(1+$ $z)^{2} D_{\mathrm{A}}^{2}$ ) $\left[10^{-14} \mathrm{~cm}^{-5}\right]$, where $n_{\mathrm{e}}=1.2 n_{\mathrm{H}}$ (see Fig. $4 \mathrm{~b}$ ). The measured central electron density is approximately $10^{-3} \mathrm{~cm}^{-3}$, the lowest among the known nearby clusters (Croston et al. 2008). 

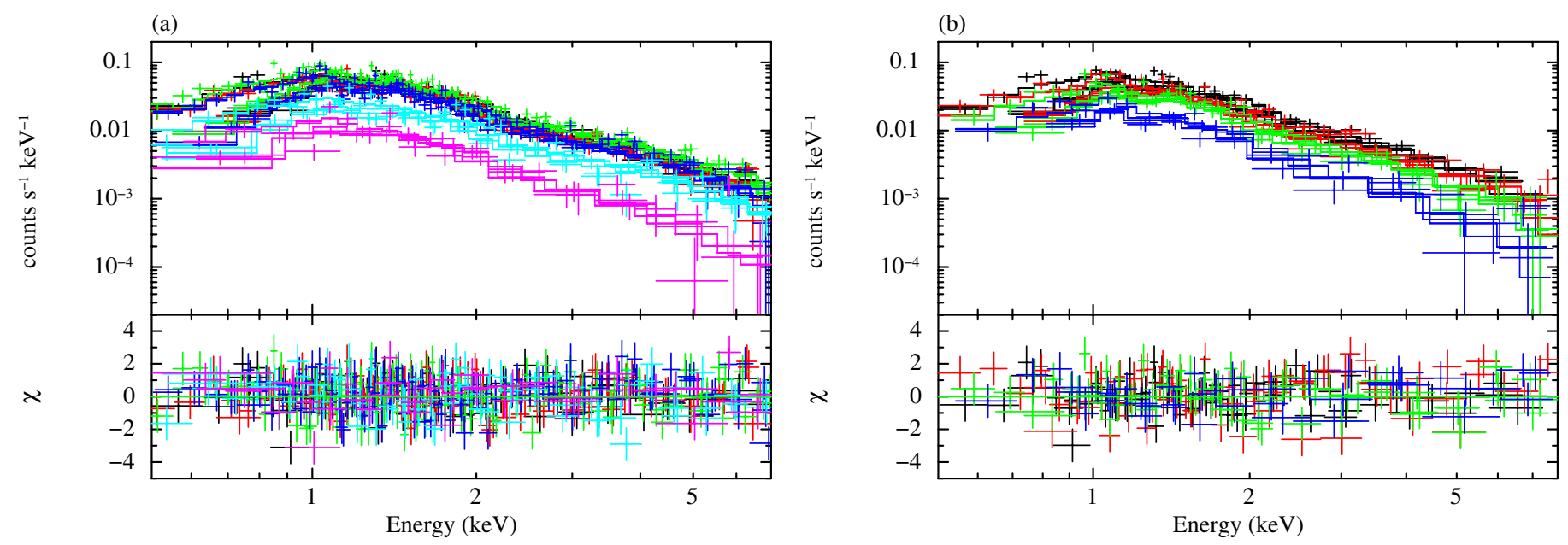

Fig. 3. Deprojection analysis of XIS spectra obtained from A76 data a) East and b) West. The crosses are the XIS-0, 1, 3 spectra accumulated from $0^{\prime}<r<3^{\prime}$ (black), $3^{\prime}<r<6^{\prime}$ (red), $6^{\prime}<r<9^{\prime}$ (green), $9^{\prime}<r<12^{\prime}$ (blue), $12^{\prime}<r<15^{\prime}$ (cyan), $15^{\prime}<r<18^{\prime}$ (magenta).

Table 4. APEC model parameters for the annular regions in A76 East and West.

\begin{tabular}{lcccccccc}
\hline \hline & \multicolumn{9}{c}{ A76 East } \\
\cline { 2 - 9 } Region & $k T[\mathrm{keV}]$ & $Z$ [solar] & Norm & $\chi^{2} /$ d.o.f. & $k T[\mathrm{keV}]$ & $Z$ [solar] & Norm & $\chi^{2} /$ d.o.f. \\
\hline $0^{\prime}<r<3^{\prime}$ & - & - & - & - & $3.56_{-0.27}^{+0.30}$ & $0.28_{-0.13}^{+0.15}$ & $2.13_{-0.16}^{+0.17} \times 10^{-3}$ & $71 / 78$ \\
$3^{\prime}<r<6^{\prime}$ & $3.32_{-0.20}^{+0.25}$ & $0.21_{-0.09}^{+0.10}$ & $3.55_{-0.23}^{+0.24} \times 10^{-3}$ & $96 / 120$ & $3.55_{-0.34}^{+0.37}$ & $0.28_{-0.15}^{+0.17}$ & $4.08_{-0.31}^{+0.33} \times 10^{-3}$ & $80 / 70$ \\
$6^{\prime}<r<9^{\prime}$ & $3.57_{-0.25}^{+0.25}$ & $0.18_{-0.08}^{+0.09}$ & $3.04_{-0.17}^{+0.18} \times 10^{-3}$ & $158 / 155$ & $2.64_{-0.27}^{+0.36}$ & $0.26_{-0.13}^{+0.19}$ & $6.88_{-0.09}^{+0.10} \times 10^{-3}$ & $60 / 64$ \\
$9^{\prime}<r<12^{\prime}$ & $3.52_{+0.30}^{-0.31}$ & $0.19_{-0.10}^{+0.11}$ & $3.12_{-0.24}^{+0.21} \times 10^{-3}$ & $140 / 127$ & $2.60_{-0.44}^{+0.56}$ & $0.33_{-0.22}^{+0.39}$ & $5.34_{-1.09}^{+1.28} \times 10^{-3}$ & $31 / 36$ \\
$12^{\prime}<r<15^{\prime}$ & $3.66_{-0.41}^{+0.45}$ & $0.31_{-0.18}^{+0.22}$ & $3.95_{+0.32}^{-0.32} \times 10^{-3}$ & $90 / 82$ & $7.1_{-3.6}^{+21.9}$ & $0.24($ fix $)$ & $1.87_{-0.42}^{+0.68} \times 10^{-3}$ & $7 / 8$ \\
$15^{\prime}<r<18^{\prime}$ & $2.79_{-0.35}^{+0.57}$ & $0.24($ fix $)$ & $1.95_{+0.24}^{-0.21} \times 10^{-3}$ & $53 / 45$ & - & - & - & - \\
\hline
\end{tabular}

Table 5. APEC model parameters obtained from the deprojection analysis of A76 East and West data.

\begin{tabular}{|c|c|c|c|c|c|c|c|c|}
\hline \multirow[b]{2}{*}{ Region } & \multicolumn{4}{|c|}{ A76 East } & \multicolumn{4}{|c|}{ A76 West } \\
\hline & $k T[\mathrm{keV}]$ & $Z$ [solar] & Norm & $\chi^{2} /$ d.o.f. & $k T[\mathrm{keV}]$ & $Z$ [solar] & Norm & $\chi^{2} /$ d.o.f. \\
\hline $0^{\prime}<r<3^{\prime}$ & $4.04_{-0.76}^{+1.04 a}$ & $0.15_{-0.05}^{+0.05 a}$ & $8.99_{-0.08}^{+0.08} \times 10^{-3 a}$ & $630 / 610$ & $3.98_{-0.88}^{+1.28}$ & $0.25_{-0.08}^{+0.09}$ & $7.05_{-0.88}^{+0.90} \times 10^{-4}$ & $299 / 255$ \\
\hline $3^{\prime}<r<6^{\prime}$ & $3.00_{-0.39}^{+0.46}$ & & $2.61_{-017}^{+0.08} \times 10^{-3}$ & & $4.00_{-0.59}^{-0.88}$ & & $1.34_{-0.30}^{-0.30} \times 10^{-3}$ & \\
\hline $6^{\prime}<r<9^{\prime}$ & $4.37_{-0.65}^{+1.00}$ & & $2.22_{-0.17}^{+0.19} \times 10^{-3}$ & & $4.00_{-0.59}^{+0.37 b}$ & & $5.70_{-0.63}^{+0.06} \times 10^{-3}$ & \\
\hline $9^{\prime}<r<12^{\prime}$ & $1.67_{-0.35}^{-0.03}$ & & $2.55_{-0.04}^{+0.03} \times 10^{-3}$ & & $2.30_{-0.25}^{+0.39}$ & & $1.12_{-0.10}^{+0.03} \times 10^{-2}$ & \\
\hline $12^{\prime}<r<15^{\prime}$ & $6.76_{-1.36}^{+1.97}$ & & $4.66_{-0.04}^{+0.04} \times 10^{-3}$ & & - & - & -0.10 & \\
\hline $15^{\prime}<r<18^{\prime}$ & $2.52_{-0.37}^{+0.50}$ & & $5.40_{-0.04}^{+0.05} \times 10^{-3}$ & & - & - & - & \\
\hline
\end{tabular}

Notes. ${ }^{(a)} 0^{\prime}<r<3^{\prime}$ spectra taken from the A76 West pointing were used in the deprojection analysis. ${ }^{(b)}$ Linked to the value for $3^{\prime}<r<6^{\prime}$.

The gas entropy, defined as $S \equiv k T n_{\mathrm{e}}^{-2 / 3}$ (Voit 2005), is evaluated from the above quantities within $0.6 r_{200} / 0.4 r_{200}$ for A76 East/West (Fig. 4c). The entropy profile is found to be flat for $r<0.2 r_{200}$ though large scatter occurs at larger radii. A very high entropy of $\sim 400 \mathrm{keV} \mathrm{cm}^{2}$ is revealed at the cluster center.

As discussed in Sect. 4, the entropy at the cluster center is of particular interest. Therefore, to check the robustness of the entropy measurement, we consider possible systematic errors due to the following three items: (i) the uniform X-ray surface brightness assumption; (ii) the background model; and (iii) the effect of the XRT's point spread function (PSF). To assess the impact of (i), we repeated the spectral analysis using arf files created by assuming a uniform cluster surface brightness instead of the observed XMM-Newton image. Although the analysis increased the statistical uncertainty in the central entropy, the result agrees with the results listed in Table 6. For (ii), the impact of background uncertainty mentioned in Sect. 2 was examined by intentionally changing the background intensity by $\pm 10 \%$. We find that this does not significantly influence the results. In assessing the effects of (iii), the width of the annular regions were made larger than the typical PSF of the Suzaku XRT $\left(\sim 2^{\prime}\right)$ to avoid significant photon mixing between adjacent regions. The xissim raytracing simulations (conducted as described in Sato et al. 2007) confirmed that the PSF effect is as weak as $\sim 20 \%$ (in terms of fraction of photons or spectral normalization factor). Accordingly, the systematic error in the central entropy is estimated to be approximately $10 \%$, which is smaller than the statistical uncertainty.

\section{Discussion}

The temperature, gas density, and entropy profiles derived from Suzaku data were well constrained out to $0.6 r_{200}$ and $0.4 r_{200}$ in the A76 East and West directions, respectively. In this section, the entropy profile of A76 is compared with those of nearby 

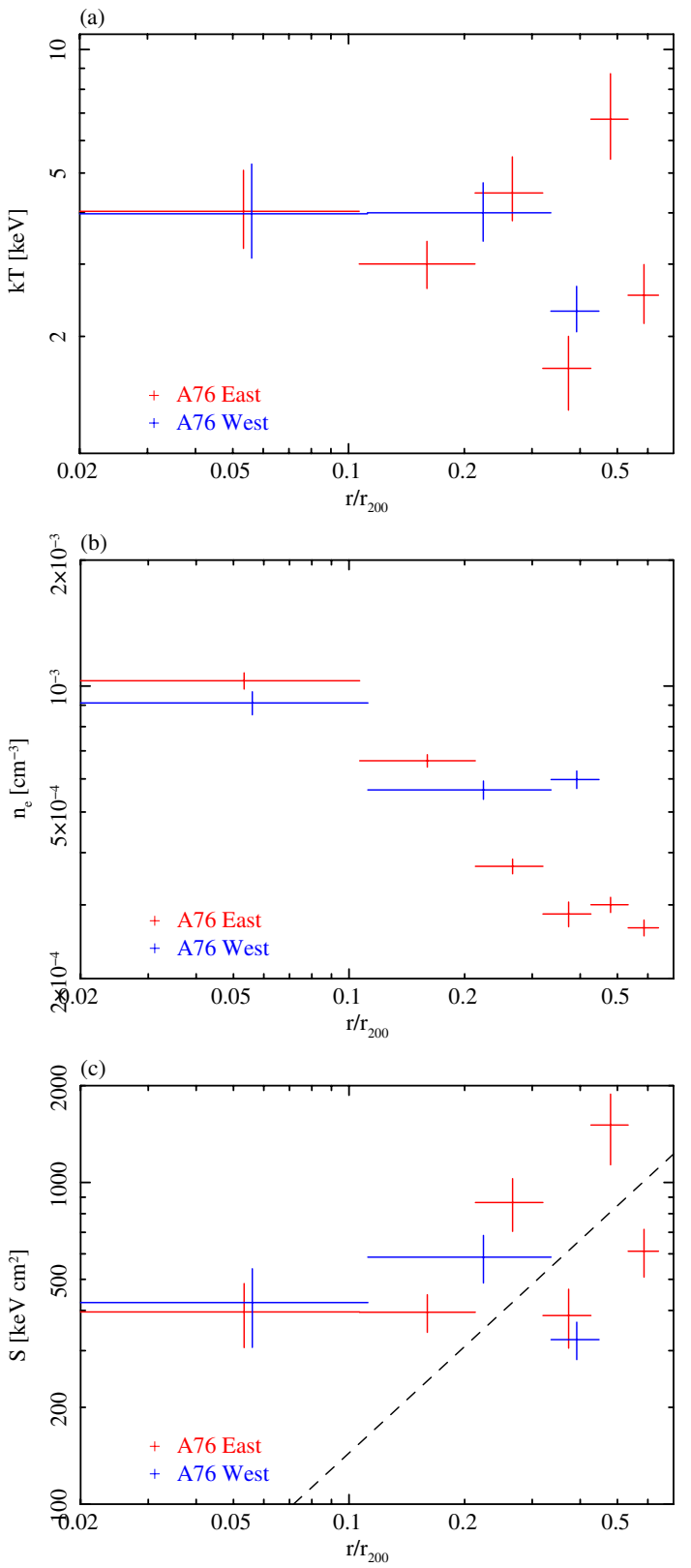

Fig. 4. a) Temperature; b) electron density; and c) entropy profiles derived from the deprojection analysis. The results for A76 East and West data are shown as red and blue crosses (the markers for A76 West are shifted horizontally by $5 \%$ for clarity). In panel c), the dashed line represents the baseline entropy profile, $S \propto r^{1.1}$ (Voit et al. 2005).

clusters and theoretical predictions, and the origin of high entropy in the LSB cluster A76 is discussed.

\subsection{Comparison of A76 entropy profile with those of other clusters}

Pratt et al. (2010) derived the entropy profiles of the REXCESS sample from XMM-Newton data. Comparing our results with theirs (Fig. 5a), we find that A76 exhibits one of the highest entropies at $r \lesssim 100 \mathrm{kpc}$. Comparison with other nearby clusters observed by Suzaku (for review, see Reiprich et al. 2013) yields similar results. On the other hand, the entropy increase at larger radii follows the overall trend of the REXCESS sample though the A76 data show significant scatter.

For a more quantitative analysis of the central entropy, Fig. $5 \mathrm{~b}$ plots the mean gas temperature of the A76 East and
West data (Table 3) and the entropy at $r<3^{\prime} \sim 0.1 r_{200}$ on the $S-T$ plane. The mean $S-T$ relations at two different radii, $0.1 r_{200}$ and $0.2 r_{200}$, derived from ten morphologically relaxed clusters using XMM-Newton are also plotted (Pratt et al. 2006). This figure clearly shows that the central entropy in A76 is significantly higher than expected for the same temperature at $0.1 r_{200}$.

\subsection{Comparison with the baseline profile and possibility of preheating and AGN feedback}

Voit et al. (2005) conducted simulations on gravitational heating and report that the radial gas entropy profile in clusters tends to follow a power law, $S(r)=550 \mathrm{keV} \mathrm{cm}^{2}\left(T_{200} / 1 \mathrm{keV}\right) r^{1.1}$, which can be used as a baseline for assessing the impact of nongravitational processes in the ICM. Figure $4 \mathrm{c}$ compares the observed A76 profile with the baseline profile calculated for $k T_{200}=$ $3.3 \mathrm{keV}$. At $0.1 r_{200}$, the observed entropy $\left(\sim 400 \mathrm{keV} \mathrm{cm}^{2}\right)$ is two times more than expected from gravitational heating alone $\left(150 \mathrm{keV} \mathrm{cm}^{2}\right)$.

Nongravitational heating is a plausible mechanism, particularly in smaller systems such as groups of galaxies. According to Ponman et al. (2003), excess entropy is on the order of $140 \mathrm{keV} \mathrm{cm}^{2}$. On the basis of Chandra analysis of 21 groups and 19 clusters, Wang et al. (2010) suggest that the entropy excess $\Delta K_{0}$ correlates with the $K$-band luminosity $L_{K}$ of the central dominating galaxies in the systems and is attributable to AGN feedback. Utilizing the $K$-band $\operatorname{luminosity} \log \left(L_{K} / L_{K, \odot}\right)=11.5$ for IC 1568 and the relationship shown by Wang et al. (2010) $\Delta K_{0} \propto L_{K}^{1.6}$, the entropy excess is estimated as $\Delta K_{0} \sim 1 \mathrm{keV} \mathrm{cm}^{2}$. Therefore, the AGN feedback, as well as preheating, is an unlikely cause of the high-entropy generation in A76.

\subsection{Implication for the origin of high entropy in A76}

In Sect. 3, the gas properties were mainly derived from the radially averaged spectra. The X-ray image, however, reveals an inhomogeneous gas distribution. This suggests that the cluster is at an early stage in its formation. The lowest gas density at a relatively high temperature of 3-4 keV indicates that gas compression due to gravitational potential confinement is lagging behind the gas heating. Given the low electron density of $10^{-4}-10^{-3} \mathrm{~cm}^{-3}$, the timescale of radiative cooling is significantly longer than the Hubble time across the cluster. At such a high central entropy level, the evolution of the gas in the deepening potential should be chiefly adiabatic. In this scenario, the entropy would remain unusually high even if the cluster existed in a relaxed state, a phenomenon not observed in other relaxed clusters. This discrepancy may be an artifact if the gas in the central region is heavily clumped. Low-entropy clumps in the high-entropy region could settle at the center as the cluster relaxes. As shown in the XMM-Newton image of Fig. 1, the ICM indeed appears clumpy, with small denser regions particularly common in the halos of several galaxies. This ICM could segregate to the center and form a lower entropy core during cluster relaxation.

A clumpy substructure is particularly apparent in the southeastern region of the cluster (hereafter, referred to as the SE blob). From the XIS spectra of the $r=5.5$ circular region enclosing the SE blob (Fig. 1), $k T$ is obtained as $3.2 \pm 0.2 \mathrm{keV}$. The density and entropy are estimated as $n_{\mathrm{e} 0}=(7.1 \pm 0.3) \times$ $10^{-4} \mathrm{~cm}^{-3}$ and $S=397 \pm 32 \mathrm{keV} \mathrm{cm}^{2}$, assuming that the emission comes from a uniform sphere of radius $r=5^{\prime} .5$. Both temperature and density are significantly high within the same radial range (0.3-0.5) $r_{200}$ in A76 East (Fig. 4), resulting in entropy 

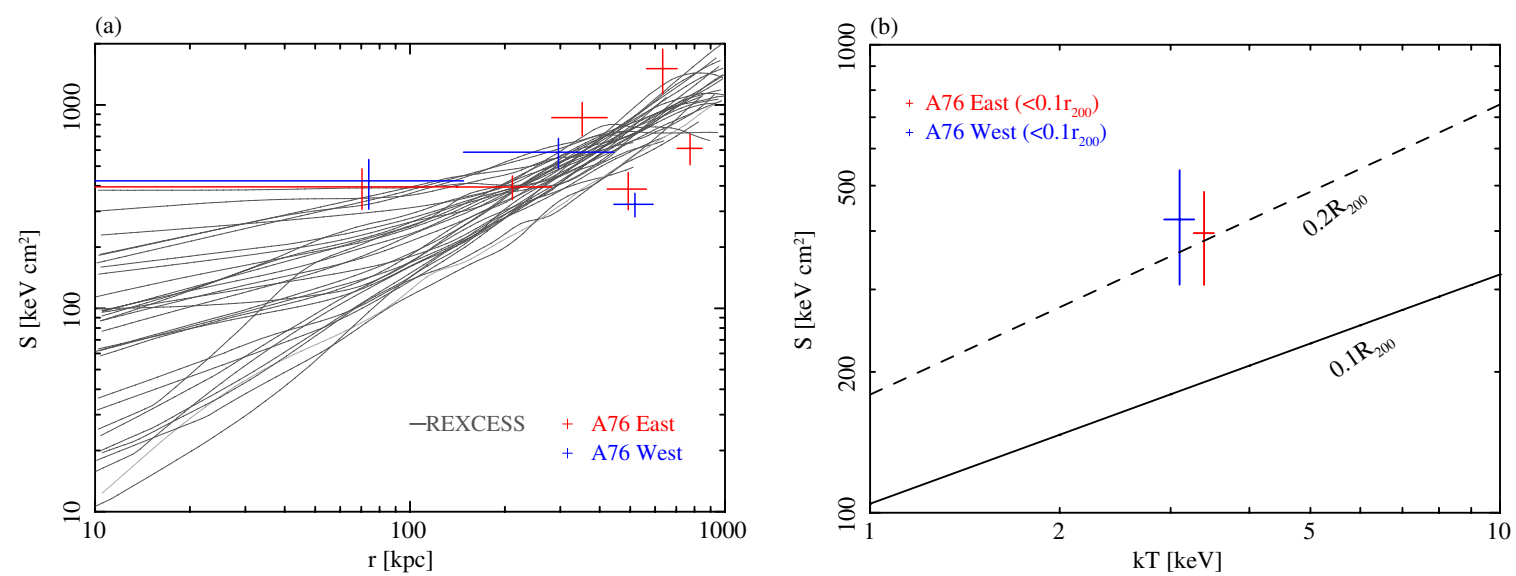

Fig. 5. a) Comparison of radial entropy profiles between A76 and the REXCESS sample (gray lines; Pratt et al. 2010). b) Correlation between entropy and temperature. The red and blue crosses show the Suzaku results for A76 East and West data, respectively, while the solid and dashed lines show the $S-T$ relationships of 10 morphologically relaxed clusters at $0.1 r_{200}$ and $0.2 r_{200}$ (Pratt et al. 2006).

Table 6. Gas density and entropy in A76 East and West.

\begin{tabular}{lcccc}
\hline \hline \multirow{2}{*}{ Region } & \multicolumn{2}{c}{ A76 East } & \multicolumn{2}{c}{ A76 West } \\
\cline { 2 - 5 } & $n_{\mathrm{e} 0}\left[\mathrm{~cm}^{-3}\right]$ & $S\left[\mathrm{keV} \mathrm{cm}^{2}\right]$ & $n_{\mathrm{e} 0}\left[\mathrm{~cm}^{-3}\right]$ & $S\left[\mathrm{keV} \mathrm{cm}^{2}\right]$ \\
\hline $0^{\prime}<r<3^{\prime}$ & $(1.03 \pm 0.05) \times 10^{-3}$ & $395 \pm 89$ & $(9.11 \pm 0.58) \times 10^{-4}$ & $423 \pm 116$ \\
$3^{\prime}<r<6^{\prime}$ & $(6.63 \pm 0.23) \times 10^{-4}$ & $394 \pm 52$ & $(5.65 \pm 0.28) \times 10^{-4 a}$ & $585 \pm 98^{a}$ \\
$6^{\prime}<r<9^{\prime}$ & $(3.71 \pm 0.15) \times 10^{-4}$ & $866 \pm 161$ & & \\
$9^{\prime}<r<12^{\prime}$ & $(2.85 \pm 0.19) \times 10^{-4}$ & $385 \pm 80$ & $(5.98 \pm 0.29) \times 10^{-4}$ & $324 \pm 43$ \\
$12^{\prime}<r<15^{\prime}$ & $(3.00 \pm 0.13) \times 10^{-4}$ & $1508 \pm 373$ & - & - \\
$15^{\prime}<r<18^{\prime}$ & $(2.65 \pm 0.12) \times 10^{-4}$ & $611 \pm 103$ & - & - \\
\hline
\end{tabular}

Notes. ${ }^{(a)}$ Values obtained from region $3^{\prime}<r<9^{\prime}$ in A76 West.

comparable to what is obtained at the same radius from the cluster center in other directions. The above arguments and the spatial extent suggest that the SE blob is probably a group-scale system that has undergone gas heating in the cluster potential.

\section{Summary}

We have performed X-ray spectral analysis of the LSB cluster A76, which is characterized by extremely low X-ray surface brightness. We constructed the profiles of gas temperature, density, entropy from the $S u z a k u$ satellite data, and out to large radii $\left(0.6 r_{200}\right.$ in A76 East and $0.4 r_{200}$ in A76 West data) for the first time. The central gas entropy $\left(\sim 400 \mathrm{keV} \mathrm{cm}^{2}\right)$ is one of the highest among the known nearby clusters and significantly higher than what is predicted from the mean $S-T$ relationship. Given that the gas density is extremely low for the observed high temperature of 3-4 keV, and because the gas distribution in the cluster is irregular and clumpy, we suggest that A76 is at an early stage of cluster formation and that gas compression caused by potential confinement is lagging behind the gas heating. Currently A76 is the only LSB cluster to be analyzed using Suzaku data. To further clarify the nature of LSB clusters and their thermodynamic evolution, we consider that many more galaxy clusters must be sampled and analyzed.

Acknowledgements. We are grateful to the Suzaku team members for satellite operation and instrumental calibration. We would also like to thank G. W. Pratt for supplying the entropy profiles for the REXCESS sample and K. Sato for suggesting a useful means of calculating the PSF effect. This study is in part supported by a Grant-in-Aid by the Ministry of Education, Culture, Sports, Science and Technology, 22740124 (NO). G.C. acknowledges support from Deutsches Zentrum für Luft und Raumfahrt with the program ID 50 R 1004. G.C. and H.B. acknowledge support from the DFG Transregio Program TR33 and the Munich Excellence Cluster "Structure and Evolution of the Universe".

\section{References}

Anders, E., \& Grevesse, N. 1989, Geochim. Cosmochim. Acta, 53, 197 Arnaud, M., Pointecouteau, E., \& Pratt, G. W. 2005, A\&A, 441, 893 Böhringer, H., Schuecker, P., Guzzo, L., et al. 2001, A\&A, 369, 826 Böhringer, H., Schuecker, P., Pratt, G. W., et al. 2007, A\&A, 469, 363 Croston, J. H., Pratt, G. W., Böhringer, H., et al. 2008, A\&A, 487, 431 Ishisaki, Y., Maeda, Y., Fujimoto, R., et al. 2007, PASJ, 59, 113

Kalberla, P. M. W., Burton, W. B., Hartmann, D., et al. 2005, A\&A, 440, 775 Koyama, K., Tsunemi, H., Dotani, T., et al. 2007, PASJ, 59, 23

Mitsuda, K., Bautz, M., Inoue, H., et al. 2007, PASJ, 59, 1

Neumann, D. M., \& Arnaud, M. 1999, A\&A, 348, 711

Ota, N., \& Mitsuda, K. 2004, A\&A, 428, 757

Ponman, T. J., Sanderson, A. J. R., \& Finoguenov, A. 2003, MNRAS, 343, 331 Pratt, G. W., Arnaud, M., \& Pointecouteau, E. 2006, A\&A, 446, 429

Pratt, G. W., Croston, J. H., Arnaud, M., \& Böhringer, H. 2009, A\&A, 498, 361 Pratt, G. W., Arnaud, M., Piffaretti, R., et al. 2010, A\&A, 511, A85 Reiprich, T. H., Basu, K., Ettori, S., et al. 2013, Space Sci. Rev. [arXiv: 1303.3286]

Sakuma, E., Ota, N., Sato, K., Sato, T., \& Matsushita, K. 2011, PASJ, 63, 979

Sato, K., Yamasaki, N. Y., Ishida, M., et al. 2007, PASJ, 59, 299

Serlemitsos, P. J., Soong, Y., Chan, K.-W., et al. 2007, PASJ, 59, 9

Smith, R. K., Brickhouse, N. S., Liedahl, D. A., \& Raymond, J. C. 2001, ApJ, 556, L91

Tawa, N., Hayashida, K., Nagai, M., et al. 2008, PASJ, 60, 11

Uchiyama, Y., Maeda, Y., Ebara, M., et al. 2008, PASJ, 60, 35

Uchiyama, H., Ozawa, M., Matsumoto, H., et al. 2009, PASJ, 61, 9

Voit, G. M. 2005, Rev. Mod. Phys., 77, 207

Voit, G. M., Kay, S. T., \& Bryan, G. L. 2005, MNRAS, 364, 909

Wang, Y., Xu, H.-G., Gu, J.-H., et al. 2010, Res. Astron. Astrophys., 10, 1013 